OPEN ACCESS

Edited by:

Rong-Rong $\mathrm{He}$,

Jinan University, China

Reviewed by:

Yang Chen,

Guangzhou University of Chinese

Medicine, China

Tian GuiHua,

Dongzhimen Hospital, Beijing

University of Chinese Medicine, China

*Correspondence:

Lie Yu

Ilyypk120@126.com

Hong Lu

zzu_luhong@163.com

${ }^{\dagger}$ These authors have contributed equally to this work

Specialty section:

This article was submitted to

Ethnopharmacology,

a section of the journa

Frontiers in Pharmacology

Received: 23 July 2019

Accepted: 09 March 2020

Published: 25 March 2020

Citation:

Wang S, Yu L, Sun G, LiU Y, Hu W, Liu Y, Peng T, Wang $X$, Cheng J, Sr A, Qin B and Lu H (2020) Danhong Injection Protects Hemorrhagic Brain by Increasing Peroxiredoxin 1 in Aged Rats.

Front. Pharmacol. 11:346. doi: 10.3389/fphar.2020.00346

\section{Danhong Injection Protects Hemorrhagic Brain by Increasing Peroxiredoxin 1 in Aged Rats}

\author{
Shang Wang ${ }^{1+}$, Lie Yu ${ }^{1 *}$, Guifang Sun ${ }^{1}$, Yu Liu ${ }^{1}$, Wentao Hu ${ }^{1}$, Yanru Liu ${ }^{1}$, Tao Peng ${ }^{1}$, \\ Xiaojun Wang ${ }^{1}$, Jingliang Cheng ${ }^{2}$, Aravintakumar $\mathrm{Sr}^{1}$, Bo Qin ${ }^{3}$ and Hong $\mathrm{Lu}^{1 *}$ \\ ${ }^{1}$ Department of Neurology, The First Affiliated Hospital of Zhengzhou University, Zhengzhou, China, ${ }^{2}$ Department of \\ Magnetic Resonance, The First Affiliated Hospital of Zhengzhou University, Zhengzhou, China, ${ }^{3}$ Translational Medicine \\ Centre, The First Affiliated Hospital of Zhengzhou University, Zhengzhou, China
}

Intracerebral hemorrhage $(\mathrm{ICH})$ is a severe cerebrovascular disease with a high incidence, mortality and disability rate. Danhong injection (DHI) is beneficial for ischemic stroke, but is prohibited for $\mathrm{ICH}$ due to risk of bleeding. The present study aims to explore the potential therapeutic time window and molecular mechanism of $\mathrm{DHI}$ in a collagenase-induced ICH model in aged rats. $\mathrm{DHI}$ administration after $\mathrm{ICH}$ could significantly improve body weight and neurological deficits, and reduce the hematoma volume and brain water content when compared to the vehicle control. Furthermore, the protective effect of $\mathrm{DHI}$ administration on days 1-3 after $\mathrm{ICH}$ was superior to those on days 3-5 or 7-9 after $\mathrm{ICH}$. DHI remarkably increased the Peroxiredoxin 1 (Prx1) expression in astrocytes and reduced the expression of inflammatory factors tumor necrosis factor- $\alpha$ (TNF- $\alpha$ ) and interleukin- $\beta(\mathrm{IL}-1 \beta)$ after $\mathrm{ICH}$. The immediate treatment of Prx1 inhibiter chelerythrine (Che) after $\mathrm{ICH}$ abolished the protective effect of $\mathrm{DHI}$. Furthermore, the Che treatment reduced the expression of Prx 1 in astrocytes, but increased the expression of TNF- $\alpha$ and $I \mathrm{~L}-1 \beta$ after ICH. DHI treatment could not reverse these changes. Therefore, the earlier DHI is administered, the better the neuroprotective effect. $\mathrm{DH}$ exerts antioxidative and antiinflammatory function by increasing Prx 1 in astrocytes. These present results may change the established understanding of $\mathrm{DH}$, and reveal a novel treatment approach for $\mathrm{ICH}$.

Keywords: aged rat, Danhong injection, intracerebral hemorrhage, hematoma volume, Peroxiredoxin 1

\section{HIGHLIGHTS}

- DHI may be used to treat intracerebral hemorrhage.

- The earlier DHI is administered, the better the neuroprotective effect.

- DHI exerts antioxidative and anti-inflammatory function by increasing $\operatorname{Pr} x 1$ in astrocytes.

\footnotetext{
Abbreviations: ICH, intracerebral hemorrhage; DHI, Danhong injection; Prxl, Peroxiredoxin 1; Che, chelerythrine; mNSS, modified neurologic severity score; PBS, phosphate-buffered saline; MRI, magnetic resonance imaging; $\mathrm{H}_{2} \mathrm{O}_{2}$, hydrogen peroxide; Mst, mammalian sterile twenty; GFAP, glial fibrillary acidic protein.
} 


\section{INTRODUCTION}

Intracerebral hemorrhage $(\mathrm{ICH})$ is a catastrophic disease that causes severe disability and high mortality in adults. The 1month mortality rate of $\mathrm{ICH}$ patients can reach up to $40 \%$, although significant progress has been made in clinical treatment (Cordonnier et al., 2018; Hemphill et al., 2018). The cascading events induced by ICH are the main cause of secondary damage (Ironside et al., 2019). Oxidative stress and inflammation have been recognized as the major disruptive factors after ICH (Wang, 2010; Hu et al., 2016). Furthermore, these two are closely correlated. Oxidative stress can mediate the expression of proinflammatory cytokines such as tumor necrosis factor- $\alpha$ (TNF- $\alpha$ ) and interleukin- $\beta$ (IL- $\beta$ ), while proinflammatory cytokines can upregulate the production of reactive oxygen species (ROS). Therefore, approaches that could inhibit oxidative stress and/or inflammation can reduce hematoma volume and promote neurological recovery after ICH (Wang et al., 2018).

Danhong injection (DHI) is a traditional Chinese medicine extracted from two herbs Salviae miltiorrhiza Bunge (Danshen, China) and Carthamus tinctorius L (Honghua, China). The highperformance liquid chromatography (HPLC) analysis revealed that the main components of DHI are flavonoids and phenolic compounds, such as tanshinone, tanshinol acid, salvianolic acid $\mathrm{B}$, protocatechuic aldehyde, rosmarinic acid, and hydroxysafflor yellow A (Liu et al., 2013; Li et al., 2017). The quality control of DHI is strictly according to the standard of the China Food and Drug Administration (CFDA), and fingerprint technology has been adopted in the process of production to ensure its quality (SFDA, 2002). DHI has been considered to accelerate blood circulation and remove blood stasis (Zhang et al., 2016). Hence, this has been widely used in Chinese clinical practice for treating cardiovascular and cerebral occlusive diseases (Chen et al., 2011), such as myocardial and cerebral ischemia injury, but is prohibited for ICH treatment according to its instruction for use (Guan et al., 2013; Guo et al., 2015). Its remarkable neuroprotective effects are mainly attributed to the antioxidative and anti-inflammatory function of DHI (Sun et al., 2009; Wang et al., 2016; Lyu et al., 2017). Therefore, the investigators explored whether DHI could be used to treat ICH due to its strong antioxidative and anti-inflammatory effects.

Peroxiredoxins (Prxs) is a ubiquitous family of antioxidant enzymes, which plays a dominant role in regulating the level of peroxides within cells and in protecting neurons from oxidative insult (Perkins et al., 2015). Recent studies have revealed the additional functions of Prxs in stress-induced gene expression and inflammation-related biological reactions, such as tissue repair and parasite infection. Notably, $\operatorname{Pr} x 1$ is the most abundant subtype in mammals. This belongs to the 2-Cys Prxs subfamily, which is a homodimer in cytosol and utilizes thioredoxin 1 as an electron donor to directly convert hydrogen peroxide $\left(\mathrm{H}_{2} \mathrm{O}_{2}\right)$ into $\mathrm{H}_{2} \mathrm{O}$ (Rhee et al., 2005; Ledgerwood et al., 2017). Mammalian sterile twenty (Mst)1, which is a serine/ threonine protein kinase, can be activated by cellular stressors including $\mathrm{H}_{2} \mathrm{O}_{2}$, and Mst1 inactivates Prxl by phosphorylating it at Thr-90 and Thr-183 (Rawat et al., 2013). Chelerythrine (Che) is a special agonist for Mst1 (Yamamoto et al., 2003). Increasing the level of Mstl can specifically induce the phosphorylation of Prx1 leading to the inactivation of its biological function. The phosphorylation of Prx1 could not be detected though the anti$\operatorname{Prx} 1$ antibody.

The present study attempted to explore the potential therapeutic time window and underlying mechanism of DHI for treating $\mathrm{ICH}$. These results would provide a theoretical foundation and a novel strategy for ICH clinical treatment with DHI.

\section{MATERIALS AND METHODS}

\section{Animals}

The present study was carried out according to the recommendations of the Institutional Animal Care and Use Committee, and approved by the Ethics Committee of Zhengzhou University. Adult male Sprague-Dawley (SD) rats, weighing 600-700 g (18 months old) (Masoro, 1980), were purchased from the Animal Center of Henan province, and subjected to ICH. Animals were individually fed and kept in cages at $22^{\circ} \mathrm{C} \pm 2{ }^{\circ} \mathrm{C}$, with a relative humidity of $50 \% \pm 10 \%$ and a 12 -h light/dark cycle. A maximum of six rats were kept in a cage $\left(470 \times 300 \times 150 \mathrm{~mm}^{3}\right)$. These animals had free access to food and water.

\section{ICH Rat Model}

The ICH model was carried out based on a previous approach (Rosenberg et al., 1990). Rats were fixed in a stereotactic frame (RWD Life Science, Shen Zhen, China) after being anesthetized with $10 \%$ chloral hydrate (intraperitoneal injection). Then, 1mm craniectomy was performed and a stereo-tactical guided needle was inserted into the right striatum at the following coordinates relative to the bregma: $0.2 \mathrm{~mm}$ anterior, $3.0 \mathrm{~mm}$ lateral, and $6.0 \mathrm{~mm}$ deep. Then, $2 \mu \mathrm{l}$ of collagenase VIIs $(0.25 \mathrm{U} /$ $\mu \mathrm{l}$, Sigma-Aldrich) was injected at a stable speed of $0.2 \mu \mathrm{l} / \mathrm{min}$. In order to prevent backflow, the needle was left in place for $10 \mathrm{~min}$. Rats in the sham group received needle insertion, but without collagenase injection. After the injection, the needle was removed, the burr hole was filled with bone wax, and the wound was sutured.

\section{Magnetic Resonance Imaging}

Based on a previous study (Del Bigio et al., 1996; Yang et al., 2017), the hematoma volumes were assessed after ICH $(n=12)$ by magnetic resonance imaging (MRI), which was conducted on a 3.0-T horizontal bore magnet MRI system (General Electric, USA). A birdcage volume resonator was used to attain the radiofrequency transmission, and the signal was received via a four-element surface coil located over the head of the rat. In order to accurately position the rat inside the magnet bore, gradient-echo pilot scans were conducted at the initiation of each imaging session. The T2-weighted images were acquired using 15 consecutive slices of $2-\mathrm{mm}$ thickness. Then, the hematoma volumes were manually traced from the T2 maps, and the 
mean signal intensity was measured by two imaging analysts, who blinded to the experimental conditions. During the scanning process, each rat was covered with a quilt to maintain body temperature.

\section{Drug Administration}

The DHI (drug approval number: Z20026866; product batch number: 13062020) was provided by BuChang Pharmaceutical Co. Ltd. (HeZe, Shandong, China), and prepared according to the statement of the CFDA. Briefly, Radix et Rhizoma Salviae Miltiorrhizae $(750 \mathrm{~g})$ was powdered and infused into $30 \%$ ethanol $(7.5 \mathrm{~L})$ for $1 \mathrm{~h}$ at $50^{\circ} \mathrm{C}$ for two times. Then, the extract was filtrated and $250 \mathrm{~g}$ of Flos Carthami was added into the residue. Afterwards, the mixture was immersed into $2.5 \mathrm{~L}$ of water for $1 \mathrm{~h}$ at $35^{\circ} \mathrm{C}$ for two times. The water extract was mixed with the alcoholic extract, and vacuum evaporated to a relative density of $1.10-1.20\left(65^{\circ} \mathrm{C}\right)$. Then, in order to obtain the isotonic solution with a $\mathrm{pH}$ value of $6-7$, the appropriate sodium chloride and sodium hydroxide for injection were added. The solution was filtered and stored at $4^{\circ} \mathrm{C}$ for $24 \mathrm{~h}$. Afterwards, water was added for injection up to $1.0 \mathrm{~L}$, and the liquid was filtered again, sterilized, and encapsulated into ampoules. Thus, the DHI was obtained (SFDA, 2002). The HPLC analysis revealed that the main components of the DHI were tanshinone, tanshinol acid, salvianolic acid B, protocatechuic aldehyde, rosmarinic acid, and hydroxysafflor yellow A (Jiang et al., 2015). The quality control standard for the DHI according to the National Drugs Surveillance Administrative Bureau is that the total amount of danshensu (molecular formula: $\mathrm{C} 9 \mathrm{H} 10 \mathrm{O} 5$ ) and protocatechuic aldehyde (molecular formula: $\mathrm{C} 7 \mathrm{H} 6 \mathrm{O} 3$ ) should not be lower than $0.5 \mathrm{mg}$ in $1 \mathrm{ml}$ of injection, as analyzed by HPLC (He et al., 2012).

A commonly used dosage of DHI in ischemic stroke of a previous study was converted (Guo et al., 2015), and the clinical practice dose $(1.0 \mathrm{ml} / \mathrm{kg}$. d) was chosen for the present study. These animals were randomly divided into three groups (Han et al., 2019), as shown in Figure 1: (1) sham-operated (sham) group $(\mathrm{n}=36),(2) \mathrm{ICH}+$ vehicle group $(\mathrm{n}=36)$, and (3) $\mathrm{ICH}+$ DHI group $(n=36)$. Animals in the $\mathrm{ICH}+\mathrm{DHI}$ group were intraperitoneally administered with DHI on days 1, 3, and 7 after $\mathrm{ICH}(\mathrm{n}=12)$. For animals in the sham and ICH + vehicle group, equal volumes of saline were administered in the abdominal cavity.

In order to explore the underlying mechanism of DHI treatment in ICH, the MST1 agonist, Che was dissolved in dimethyl sulfoxide (DMSO, $10 \mu \mathrm{l}, 1 \mathrm{mmol} / \mathrm{L}$ ) (Sacchetti and Bielavska, 1998). Then, rats were further randomly divided into three groups: (4) $\mathrm{ICH}+$ Che group ( $\mathrm{n}=12),(5) \mathrm{ICH}+\mathrm{DMSO}+$ DHI group $(n=12)$, and (6) ICH + Che + DHI group $(n=12)$. Che was administered to the lesion core immediately after $\mathrm{ICH}$, and DMSO was administered as the vehicle control. The DHI was continuously given at 1-3 days after ICH for rats in the $\mathrm{ICH}+\mathrm{DMSO}+\mathrm{DHI}$ and $\mathrm{ICH}+\mathrm{Che}+\mathrm{DHI}$ groups.

\section{ASSESSMENT OF ICH OUTCOME}

\section{Mortality, Body Weight, and Behavior Experiments After ICH}

The body weight and neurologic deficits (through the 14-point modified neurological severity score (mNSS), corner turn test, and tape removal task) (Schaar et al., 2010; Lekic et al., 2012; Zhu et al., 2018) of rats were evaluated on days 1, 3, 7, and 14 after $\mathrm{ICH}$. All behavior tests were performed in a blinded manner.

\section{Cresyl Violet Staining}

At 14 days after ICH induction, each group of rats were given an MRI scan. Afterwards, rats (six rats in each group) were anesthetized and intracardially perfused with phosphatebuffered saline ( $\mathrm{pH} 7.4)$, followed by $4 \%$ paraformaldehyde. Then, the brains were removed and immersed in $4 \%$ paraformaldehyde for $24 \mathrm{~h}$, and were dehydrated with $30 \%$ sucrose solution for $3-5$ days and at $4^{\circ} \mathrm{C}$. Subsequently, the

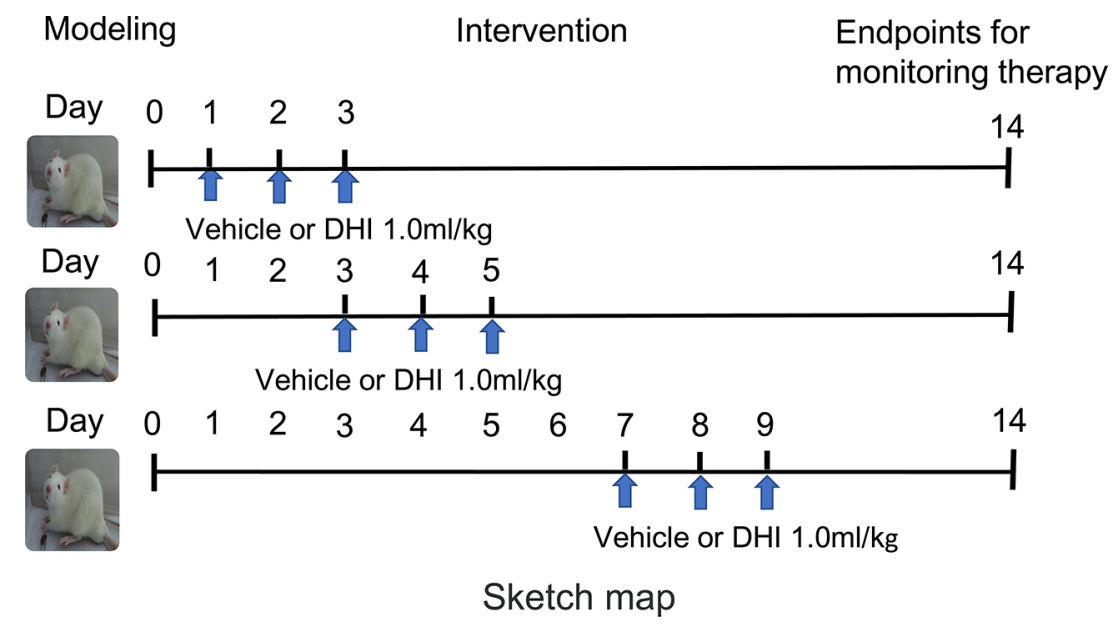

FIGURE 1 | A sketch map of the experiment schedule for the study. 
brain samples were frozen and cut into coronal frozen slices (thickness: $20 \mu \mathrm{m}$ ) using a cryostat microtome (Leica CM3050S3-1-1, Bannockburn, IL). Then, the cryosections with cresyl violet to quantify the hematoma volume (Lan et al., 2017). The damaged areas were evaluated at a $10 \times$ objective using the Image-J software. The total injury volume in cubic millimeters was calculated as the sum of the damaged area multiplied by the distance between sections of $120 \mu \mathrm{m}$ (Wu et al., 2017).

\section{Brain Water Content}

At 14 days after the MRI scans in each group, rats were sacrificed to obtain fresh brain samples. The wet weight was obtained using a precision scale, and the samples were dried in an oven at a $100^{\circ} \mathrm{C}$ for $48 \mathrm{~h}$. The brain water content was measured as a surrogate for brain edema (Han et al., 2016). This was calculated, as follows: [(wet weight - dry weight)/wet weight] $\times 100 \%$.

\section{IMMUNOFLUORESCENCE STAINING}

DHI was given at $24 \mathrm{~h}$ post-ICH as the following research target. Based on the established protocol (Lan et al., 2017), these sections were incubated overnight at $4^{\circ} \mathrm{C}$ with primary antibodies, including anti-Prx1 (Abcam, ab59538 1:300), antiNeuN (Abcam, ab177487 1:500), glial fibrillary acidic protein (GFAP) (Santa Cruz, sc33673 1:500), and anti-Iba-1 (Abcam, ab5076 1:500), followed by the appropriate fluorescenceconjugated secondary antibodies (Santa Cruz Biotechnology, CA, USA 1:300). Then, the sections were visualized using a fluorescence microscope (ZEISS Scope A1, ZEISS, Germany). Afterwards, the number of double-labeled cells in the striatum around the hematoma were counted (Chang et al., 2014). Brain sections with similar lesion areas were selected. Cell counts and the co-localization of Prx1 with NeuN/GFAP/Iba-1 were analyzed using the Image-J software (1.4, NIH). Positive cells at $40 \times 10$ magnification from five optical fields in three sections per animal were averaged. The cell densities per square millimeter were calculated.

\section{Enzyme-Linked Immunosorbent Assay for Detection of Inflammatory Factors Levels}

The concentrations of TNF- $\alpha$ and IL- 6 in the brain homogenate solution of ICH rats was measured by enzyme-linked immunosorbent assay (ELISA) kits (USCN, Life Science Inc.), according to the manufacturer's protocol.

\section{Statistical Analysis}

All data were presented as mean \pm standard deviation (SD), and SPSS 21.0 was used for all statistical analyses. First, all data were tested for normality of distribution with the Shapiro-Wilk test or Kolmogorov-Smirnov test. Then, the three groups comparison of different time points with normal distribution were compared using two-way analysis of variance (ANOVA), and the MannWhitney $\mathrm{U}$ test was used for nonparametric data. $P<0.05$ was considered statistically significant.

\section{RESULTS}

\section{DHI Promotes Body Weight and Neurological Functional Recovery After ICH}

Among the 211 rats, 11 rats died during the experiment. No death occurred in the sham group. The mortality rate in the vehicle and DHI treated group was $12.20 \%$ (5/41) and 14.29\% (6/ $42)$, respectively. However, there was no difference in mortality between the vehicle-treated and DHI-treated groups. The body weight and neurological function of vehicle-treated rats significantly decreased during the first three days after $\mathrm{ICH}$, when compared to sham operated rats $(P<0.001)$, and these gradually increased within $7-14$ days after ICH $(P<0.005)$. Rats treated with DHI on days 1-3, 3-5, or 7-9 after ICH exhibited a significantly less body weight loss (Figures 2A-C), when compared to vehicle-treated rats $(P<0.05)$. The administration of DHI on days $1-3,3-5$, or $7-9$ after ICH also significantly reduced the mNSS, (Figures 2E-G) the percentage of left turns in the corner turn test (Figures $\mathbf{3 A}-\mathbf{C}$ ), and the adhesiveremoval time (Figures 3E-G), when compared to vehicletreated rats. Furthermore, the protective effect of DHI treatment on days 1-3 after ICH on neurological function and body weight was superior, when compared to the treatment on days $3-5$ or $7-9$ (Figures $\mathbf{2 D}, \mathbf{H}$ and $\mathbf{3 D}, \mathbf{H} ; \mathbf{P}<0.05$ ). In conclusion, the earlier the treatment of DHI, the better the ability to promote neurological function and body weight recovery.

\section{DHI Reduces Hematoma Volume and Brain Edema After ICH}

The therapeutic effect of DHI on day 14 after ICH was verified. First, MRI scanning (Figure 4A) and cresyl violet (Figure 4B) staining were performed to assess the hematoma volume. The hematoma volume in the DHI group $(1.0 \mathrm{mg} / \mathrm{kg}$. d) significantly decreased, when compared to the vehicle group (Figures $4 \mathrm{C}, \mathrm{D}$; $P<0.01)$. However, rats treated with DHI on days $1-3$ after ICH had the smaller cerebral hematoma size, when compared to rats treated with DHI, on days $3-5$ or $7-9(P<0.05)$. Furthermore, the hematoma volume of rats treated with DHI on days 3-5 after ICH was smaller, when compared to rats treated with DHI on days 7-9 (Figures 4C, D). In addition, the DHI treatment simultaneously reduced the brain water content on day 14 at post-ICH (Figure 4E, $\mathrm{n}=6$; DHI at days $1-3,72.64 \% \pm 1.82 \%$; $\mathrm{DHI}$ at days $3-5,74.44 \% \pm 1.65 \%$; DHI at days $7-9,75.02 \% \pm$ $1.06 \%$; vehicle, $78.31 \% \pm 1.63 \%$; $P<0.05)$. However, the time of treatment of DHI did not affect the reduction in encephaledema (Figure 4E, $P>0.05$ ).

\section{DHI Treatment Upregulates Prx1 Expression After ICH}

Few studies have investigated the expression of Prx 1 after $\mathrm{ICH}$. Therefore, the expression pattern of Prxl after ICH was identified. The immunofluorescence staining results revealed that the expression of Prx 1 was upregulated and peaked on day 7, and began to gradually decrease on day 14 after ICH (Figures $\mathbf{5 A - L}, \mathbf{S})$. In order to verify the source of $\operatorname{Pr} x 1$ after $\mathrm{ICH}$, the 


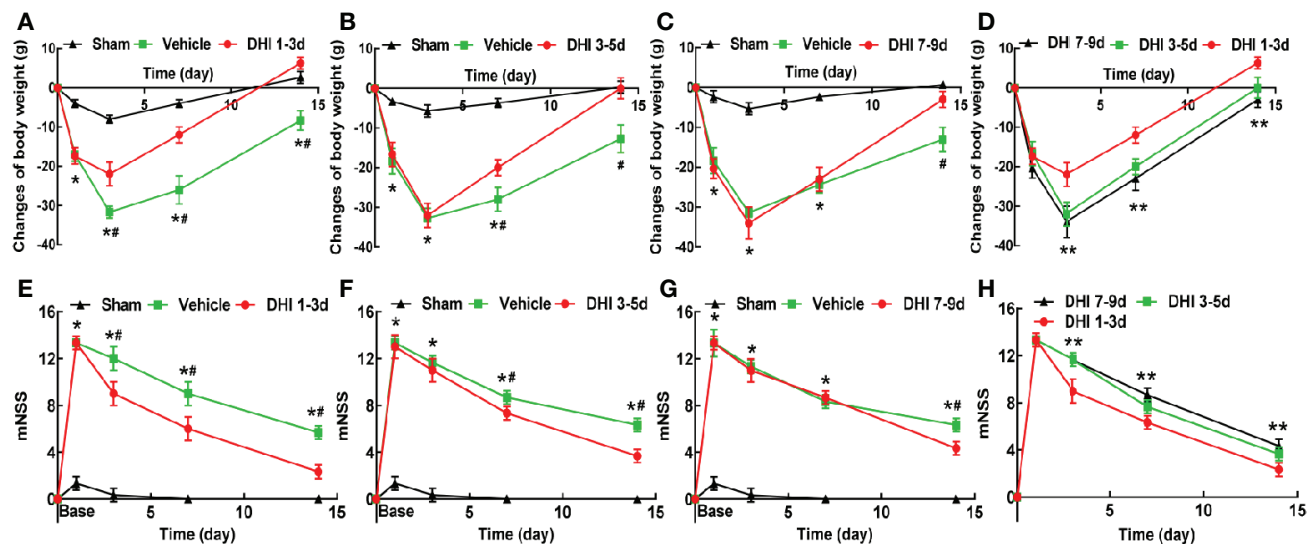

FIGURE 2 | Danhong injection (DHI) treatment promotes the recovery of body weight and neurologic function. DHI treatment significantly promotes body weight recovery (A-C) and reduces modified neurologic severity score (mNSS, E-G) after intracerebral hemorrhage (ICH). (D) The administration of DHI on days 1-3 after $\mathrm{ICH}$ exhibits greater advantage in decreasing weight loss, when compared to the administration on days $3-5$ or $7-9(\mathrm{n}=12, P<0.05)$. (H) DHI treatment on days 1-3 after $\mathrm{ICH}$ has a better effect on reducing neurological deficits, when compared to the treatment of DHI on days $3-5$ or $7-9(n=12, P<0.05)$. $\left({ }^{*} P<0.05\right.$ vs. sham; ${ }^{\#} P<0.05$ vs. vehicle, ${ }^{\star \star} P<0.05$ vs. DHI 3-5 and 7-9 days).
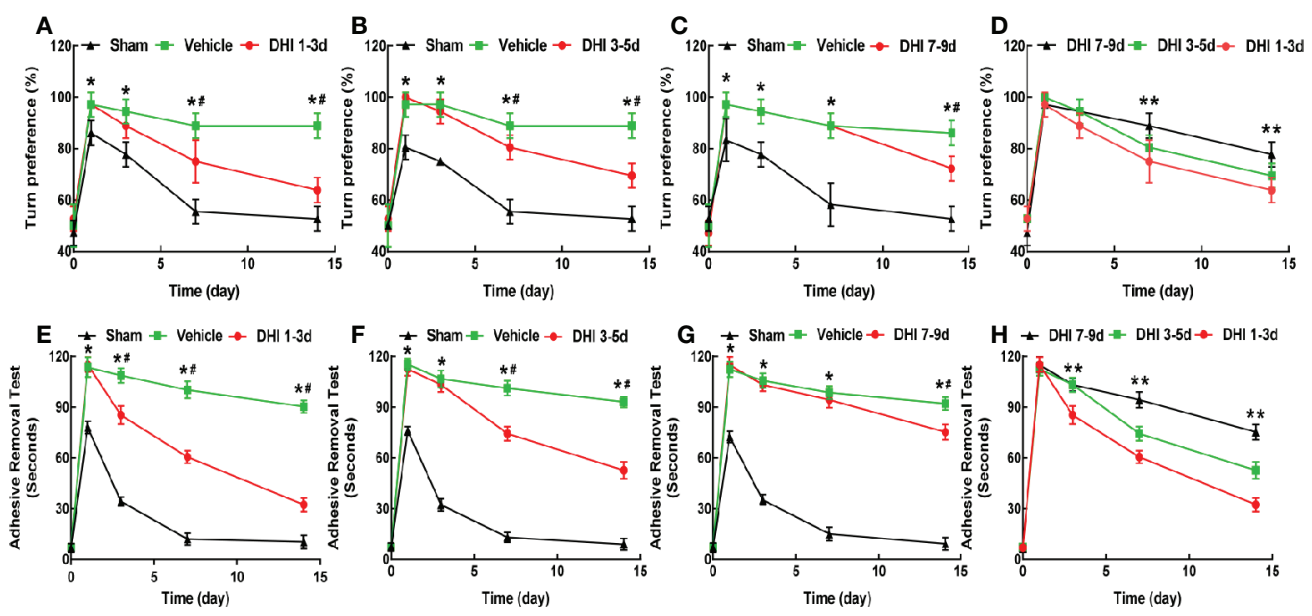

FIGURE 3 | DHI treatment improves the turn bias and somatosensory deficits. DHI treatment significantly reduces the percentage of left turns in the corner turn test (A-C) and adhesive-removal time (E-G) after ICH. (D) The DHI administration on days 1-3 after ICH has a better effect on decreasing the percentage of left turns, when compared to the administration of $\mathrm{DHI}$ on days $3-5$ or $7-9(\mathrm{n}=6, P<0.05)$. $(\mathbf{H})$ The effect of DHI treatment on days $1-3$ after ICH in reducing the adhesiveremoval time was superior to the treatment performed on days $3-5$ and $7-9(n=6, P<0.05)$. $\left({ }^{*} P<0.05\right.$ vs. sham; ${ }^{\#} P<0.05$ vs. vehicle; ${ }^{* *} P<0.05$ vs. DHI $3-5$ or 7-9 days).

expression of $\operatorname{Prx} 1$ in astrocytes (GFAP+ cells), microglias (Iba$1+$ cells), and neurons (NeuN+ cells) were assessed. The immunofluorescence staining results revealed that $\operatorname{Pr} x 1$ was mainly expressed in the cytoplasm of neuron within $24 \mathrm{~h}$ after ICH, and this gradually disappeared on day 3 (Figures $\mathbf{5 A - F}$, S). The expression of Prx1 in astrocytes was less than that in neuron within $24 \mathrm{~h}$. This dramatically increased on day 3 and peaked on day 7 after ICH (Figures 5G-L, S). However, no Prx 1 was observed to express in Iba-1+ cells (Figures $\mathbf{5 M - O}$ ). Since the neurological protective effect of the injection of DHI on days 1-3 after ICH was superior to that on days 3-5 and 7-9, the influence of DHI treatment on days 1-3 after ICH on the expression of Prx1 was tested. The immunofluorescence staining results demonstrated that $\operatorname{Pr} x 1$ was mainly expressed in astrocytes on day 3 after the DHI treatment. The DHI treatment remarkably increased the expression of $\operatorname{Prx} 1$ in astrocytes on days 3-14 after ICH, when compared to the vehicle treatment (Figures 5P-R, T; $P<0.05$ ). These results indicate that DHI mainly affects Prx1 expression in astrocytes rather than neurons. 


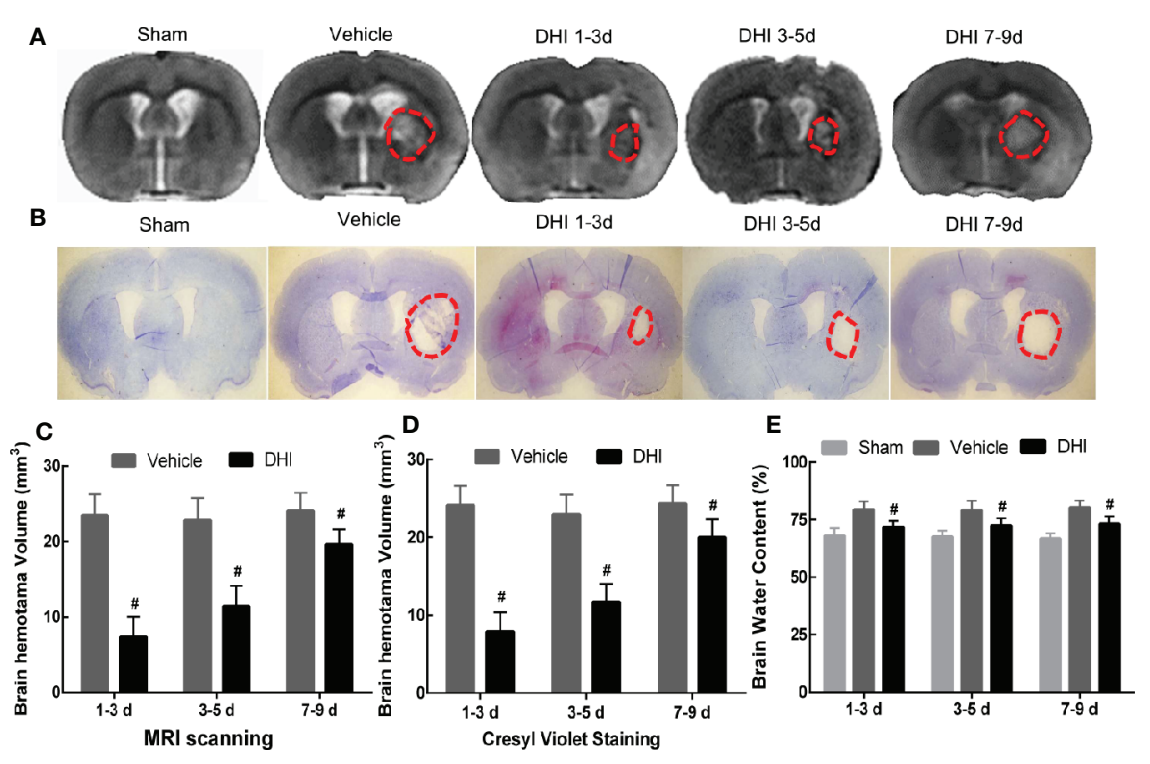

FIGURE 4 | DHI treatment reduces hematoma volume and brain water content after ICH. The (A, C) Magnetic resonance imaging (MRI) scanning and (B, D) cresyl violet staining results revealed that $\mathrm{DHI}$ treatment significantly reduced hematoma volume, when compared with the control $(n=12, P<0.01)$, and the hematoma volume in rats treated with $\mathrm{DHI}$ on days $1-3$ after $\mathrm{ICH}$ was significantly lesser, when compared to that in the other two time point groups $(\mathrm{n}=12, P<0.01)$. (E) The brain water content test revealed that the DHI treatment reduced the brain water content, when compared to the vehicle treatment $(n=6, P<0.05)$. However, the time of treatment did not affect the effect of $\mathrm{DHI}$ in reducing encephaledema $(\mathrm{n}=6, P>0.05)$. ( ${ }^{\#} P<0.05 \mathrm{vs}$. vehicle).

\section{Che Inhibits the Effects of DHI on ICH by Inactivating Prx1}

Based on the above findings, it was hypothesized that DHI exerts its neurological protective effect by upregulating the expression of Prx1. Che (a Prx1 inhibitor) could specifically mediate the phosphorylation of $\operatorname{Pr} x 1$, which leads to the inactivation of its biological function. It was found that the expression of $\operatorname{Pr} x 1$ in the $\mathrm{ICH}+\mathrm{Che}+\mathrm{DHI}$ and $\mathrm{ICH}+$ Che groups significantly decreased, when compared to the ICH group and $\mathrm{ICH}+\mathrm{DHI}$ group (Figures $\mathbf{6 A - M}, P<0.01$ ). Furthermore, there was no difference in the expression of Prx1 between the $\mathrm{ICH}+\mathrm{Che}+$ $\mathrm{DHI}$ and $\mathrm{ICH}+$ Che groups on days 3, 7, and 14 after ICH (Figures 6A-M, $P>0.05$ ). In addition, the inhibition of the biological function of Prx1 using Che before the DHI injection prevented the recovery of body weight and neurological function, and the reduction of brain water content and hematoma volume, when compared to the DHI + DMSO treatment (Figures 7A-E, $P<0.05)$.

\section{DHI Inhibits the Expression of Inflammatory Factors After ICH}

It has been reported that DHI could attenuate inflammatory reactions after ICH. Hence, the changes in TNF- $\alpha$ and IL- 6 were detected. The ELISA results revealed that DHI treatment significantly inhibited the expression of TNF- $\alpha$ and IL- 6 in $\mathrm{ICH}$ aged rats, when compared to the vehicle treatment (Figures 8A, B, $P<0.05$ ). However, with the treatment of Che after $\mathrm{ICH}$, the administration of DHI could no longer decrease the expression of TNF- $\alpha$ and IL-6 (Figures 8A, B, $P<0.05$ ).

\section{DISCUSSION}

The collagenase-induced ICH model of aging rats in the present study simulated the clinical phenomenon of spontaneous $\mathrm{ICH}$, which mainly occurs in aging adults. The present study is the first to demonstrate that DHI can be used to treat $\mathrm{ICH}$, and that this has an outstanding neuroprotective effect on ICH. Furthermore, the earlier administration of DHI led to its better ability to promote neurological function and hematoma recovery. The molecular mechanism of the neuroprotective effect of DHI involved the upregulation of $\operatorname{Pr} x 1$ and enhanced its antioxidative and anti-inflammatory functions in astrocyte. These novel findings also suggested that $\operatorname{Pr} x 1$ is a potential target to ameliorate secondary brain injury and improve longterm neurologic recovery after ICH.

A previous study revealed that aging exacerbates the astroglial reaction in response to excitotoxic damage (Castillo-Ruiz et al., 2007), increases oxidative stress, and deteriorates neurological function due to loss of neurotransmission (Monti et al., 2004). Activated glia presents with different changes in different regions in aged brain including age-related hypertrophy in the frontal cortex and a numeric increase in the hippocampus (Amenta et al., 1998). Astrocytes in aged rats also exhibit a regionspecific regulation function, which can attenuate the injury-induced cytokine response after excitotoxic damage (Campuzano et al., 2009). Given these afore-mentioned findings, it can be concluded that aged rats are different from young and adult rats when these are exposed to insults. These differences are reflected not only in its selfdefense function, but also in the process of damage (Zhao et al., 2015). Thus, aged rats were chosen as the present experimental 


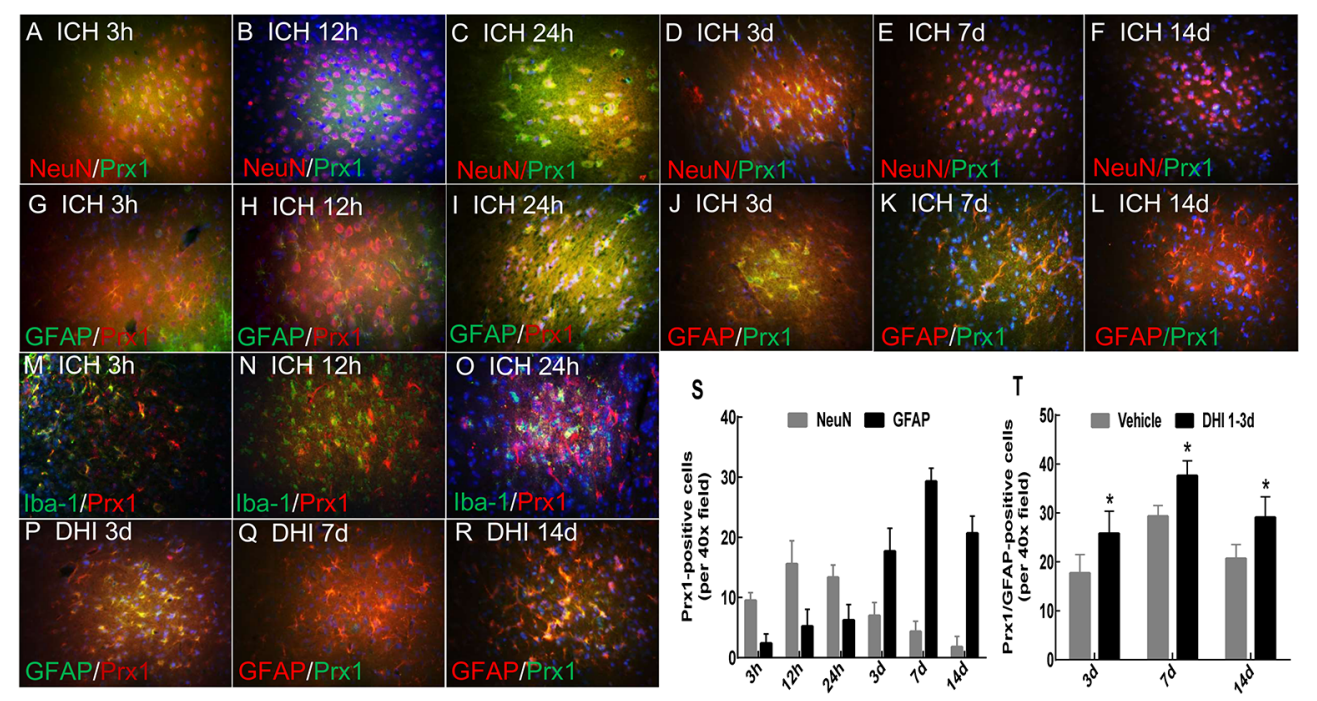

FIGURE 5 | DHI upregulates the Peroxiredoxin 1 (Prx1) expression in astrocytes after ICH. (A-L) The immunofluorescence staining results revealed that Prx 1 was mainly expressed in the cytoplasm of neurons within $24 \mathrm{~h}$ after $\mathrm{ICH}$, peaked at $12 \mathrm{~h}$, and gradually decreased (A-F). The expression of Prx 1 in astrocytes was dramatically increased and peaked on day 7 after ICH (G-L). (M-O) However, no Prx1 was observed in Iba- $1^{+}$cells. (P-R) The DHI treatment on days 1-3 after ICH remarkably increased the expression of Prx 1 in astrocytes. Majority of Prx 1 positive cells were astrocytes. (S) The quantitation of $\operatorname{Prx} 1$ expression in neurons and astrocytes after ICH. (T) The quantitation the effect of DHI treatment on days $1-3$ after $I C H$ on the Prx 1 expression in astrocytes $(n=6, P<0.05)$. (Images are shown at $400 \times$ magnification, ${ }^{*} P<0.05$ vs. vehicle).
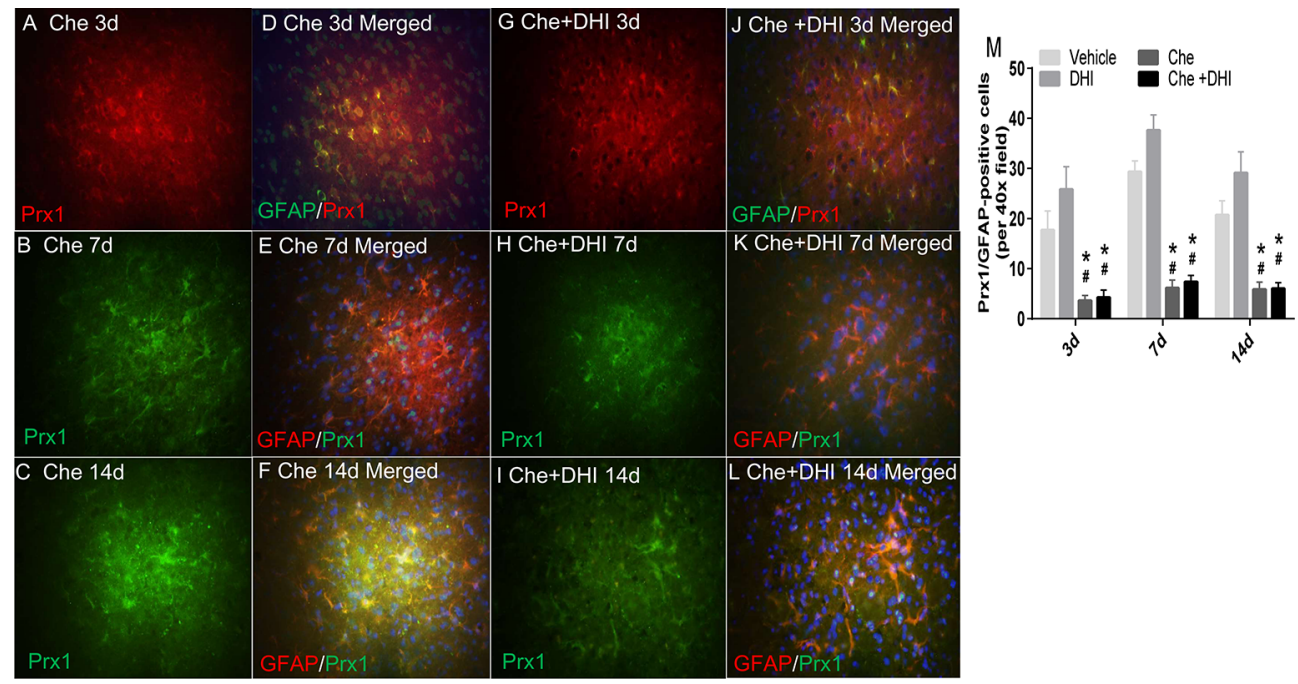

FIGURE 6 | Che inhibits the expression of Prx1 in astrocytes after ICH. (A-F) The effect of Che injection on Prx1 expression on days 3 (A, D), 7 (B, E), and 14 (C, F) after ICH. (G-L) The effect of Che + DHI treatment on Prx1 expression on days 3 (G, J), 7 (H, K), and 14 (I, L) after ICH. (M) The quantitative analysis results revealed that the immediate treatment of Che after collagenase injection significantly inhibited the expression of Prx 1 in astrocytes. DHI administration could not reverse the phenomenon. (Images are shown at 400x magnification; Che, chelerythrine; ${ }^{*} P<0.05$ vs. vehicle, ${ }^{\#} P<0.05$ vs. DHI).

objects, in order to explore the role of DHI and its potential molecular mechanism in ICH.

$\mathrm{ICH}$ is a devastating disease that brings serious burden to humans. Most patients with ICH would retain varying degrees of disability. However, drugs for treating ICH are very limited and have poor efficacy (Wang et al., 2018). DHI is a neuroprotective agent (Qian et al., 2018), and is widely used to treat cerebral (Wang et al., 2016) and myocardial ischemic diseases (Guan et al., 2013). Traditional theory deems that DHI can accelerate blood circulation and remove blood stasis. To date, it is still prohibited to use this to treat ICH due to the high risk of hemorrhage. After careful analysis of the literature 


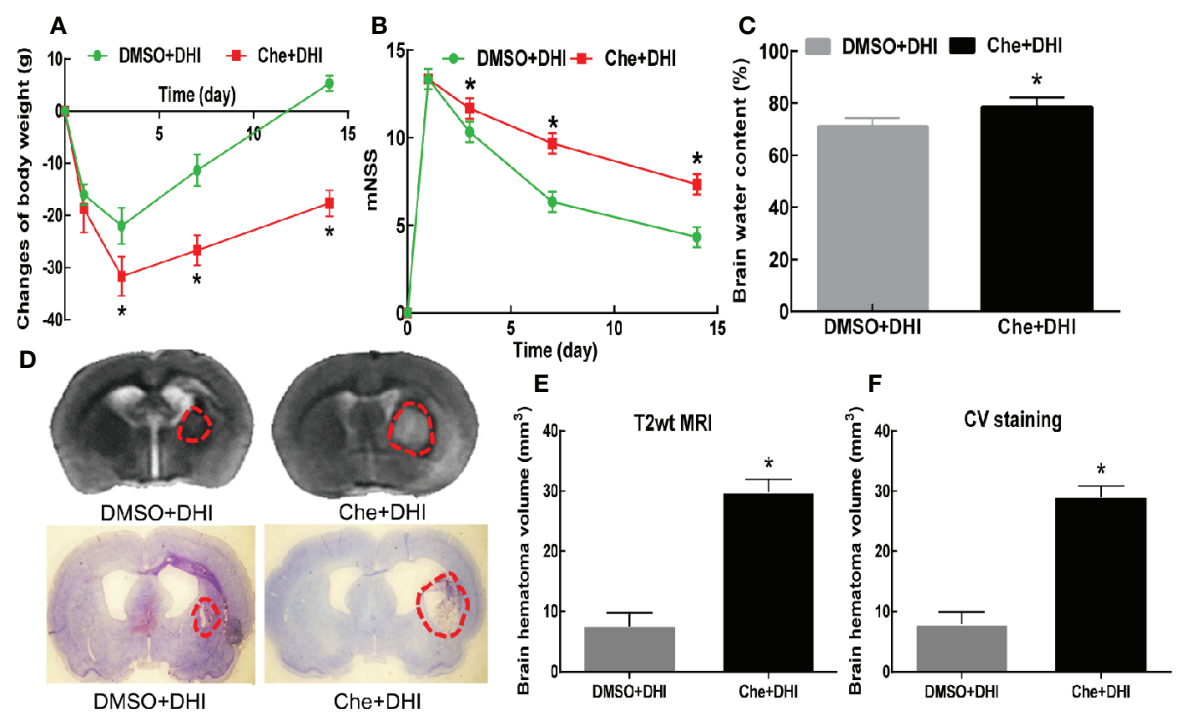

FIGURE 7 | The inactivity of Prx1 with Che after ICH abolishes the neuroprotective function of DHI. Che treatment impeded the recovery of body weight $(\mathbf{A}, \mathrm{n}=12)$ and neurological function $(\mathbf{B}, \mathrm{n}=12)$, and prevented the reduction in brain water content $(\mathbf{C}, \mathrm{n}=6)$ and hematoma volume $(\mathbf{D} / \mathbf{E} / \mathbf{F}, \mathrm{n}=6)$. ${ }^{*} P<0.05$ vs. dimethyl sulfoxide $(\mathrm{DMSO})+\mathrm{DHI}$.
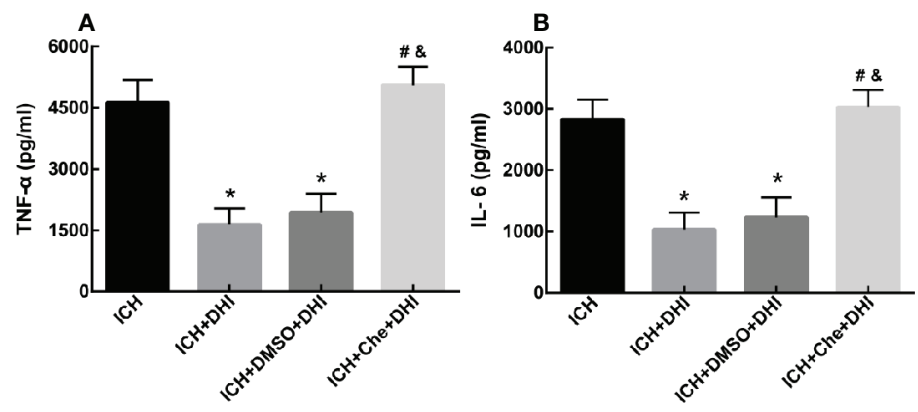

FIGURE 8 | DHI reduces the expression of inflammatory factors tumor necrosis factor- $\alpha$ (TNF- $\alpha$ ) and interleukin-6 (IL-6) after ICH. The levels of TNF- $\alpha$ and IL-6 significantly decreased in the ICH + DHI and ICH + DMSO + DHI groups, when compared to the vehicle group $(\mathbf{A}, \mathbf{B}, \mathrm{n}=6) .\left({ }^{*} P<0.05\right.$ vs. ICH). The expression of TNF- $\alpha$ and IL-6 was no longer inhibited by DHI after the injection of Che in the rat model of ICH (A, B, $n=6)$. $\left({ }^{\#} P<0.05\right.$ vs. ICH + DMSO + DHI; ${ }^{\&} P<0.05$ vs. ICH + DHI).

regarding $\mathrm{DHI}$, it was found that the most important mechanism of DHI treatment for ischemic diseases are the antioxidative stress and anti-inflammatory function. The main causes that lead to the progression of ICH are also oxidative stress and inflammation (Li et al., 2017; Feng et al., 2019). Furthermore, a previous clinical study revealed the protective effect of DHI for treating traumatic intracranial hematoma (Sun et al., 2009). Therefore, an attempt was made to explore the efficacy of DHI in treating ICH in different time windows. These above results illustrate that DHI has a neuroprotective effect for ICH. First, this did not increase motility. Second, DHI promoted the recovery of body weight and neurological function. Third, this reduced brain hemorrhagic volume and brain water content, when compared to the vehicle. Next, the dissimilitude of the protective effect of DHI treatment at different time points was assessed. It was found that there were remarkably different efficiencies among the three groups. The neuroprotective effect of DHI treatment at days 1-3 was better, when compared to the treatment on days $3-5$ or $7-9$, regardless of the recovery of weight, neurological function, or reduction of hematoma and brain edema. In conclusion, DHI can be used to treat $\mathrm{ICH}$, even in the acute phase.

A study proposed that DHI can enhance the antioxidant capacity of micro-vascular endothelial cells in the context of cerebral hypoxia (Lyu et al., 2017). In addition, DHI exerts its biological effects by changing the Nrf2 levels and upregulating the level of SOD, GSH, and MDA after ischemic stroke (Guo et al., 2014). Furthermore, the fractions of 5-7 and 17-19 in a ternary network have been demonstrated to be the main active components of DHI (Wang et al., 2016). Prxl is an antioxidative stress protein. However, its biological activity 
is inhibited by heme after ICH. Furthermore, the antioxidative function of Prx1 relays on the co-expression of HO-1 (Nakaso et al., 2000; Zhang et al., 2017). However, these studies do not depict the dynamic changes of $\operatorname{Pr} x 1$ after ICH. It was considered that scavenging $\mathrm{H}_{2} \mathrm{O}_{2}$ may be the most effective approach of DHI in exerting neuroprotection after ICH. Prxs have been shown to be the most effective protein to scavenge $\mathrm{H}_{2} \mathrm{O}_{2}$, especially $\operatorname{Prx} 1$ (Mizusawa et al., 2000). Recent evidence has indicated that $\operatorname{Pr} \times 1 / 2$ protects the brain against $\mathrm{H}_{2} \mathrm{O}_{2}$ induced apoptosis after subarachnoid hemorrhage ( $\mathrm{Lu}$ et al., 2019). Another study depicted that antioxidant Prx1 is more highly expressed than other antioxidant enzymes in monocytes and macrophages, and that $\operatorname{Prx} 1$ deficiency leads to excessive oxidative stress and impairs the maintenance of autophagic flux in macrophages (Jeong et al., 2018). Thus, Prx 1 was chosen to further determine the effect of DHI. The dynamic changes of $\operatorname{Prx} 1$ after ICH were first presented in the present study. It was found that $\operatorname{Pr} x 1$ was upregulated and peaked at $12 \mathrm{~h}$ in neuron, and on day 7 in astrocytes after ICH. A study that conducted a proteomic analysis revealed that Prx 1 was downregulated in peroxide, while the catalase expression was upregulated at $3 \mathrm{~h}$ after $\mathrm{ICH}$ (Ren et al., 2014). Another study concluded that the expression level of Prxl in the hematoma region was higher than that in other areas at 1 day after blood injection (Nakaso et al., 2000). This discrepancy may be due to the difference of the experimental objects and methods of study. Immunofluorescence staining was performed to determine the expression level and cellular location. Published literatures have focused on the integrated level of Prx1 in a whole hemorrhagic brain using the proteomic analysis method. The present results demonstrate that the expression of Prxl was further elevated by DHI especially on days 3 and 7 . The revelation of the dynamic changes of Prx 1 after ICH lays the foundation for further study.

Next, efforts were made to observe the cellular position of Prx1. A study revealed that $\operatorname{Pr} x 1$ was mainly expressed in astrocytes, and elevated after subarachnoid hemorrhage (Lu et al., 2019). Nakaso et al. elaborated that HO-1 and Prx1 were induced in reactive astrocytes (mainly at days 14 and 28) around the hemorrhagic region. However, both proteins were not induced in neurons (Nakaso et al., 2000). Another research revealed that $\operatorname{Pr} x 1$ was a major hemorrhagic stressinducible isoform of Prxs in ICH. The insult stimulated the Prx1 expression and mediated extracellular release, leading to the activation of TLR-4/NF- $\kappa \mathrm{B}$ signaling and the production of inflammatory cytokines (TNF- $\alpha$, IL-6, and IL-17) (Liu et al., 2016). The present study revealed that Prx1 was mainly expressed in neuronal cells (which peaked at $12 \mathrm{~h}$ ) in the peri-hematoma region in the first 3 days after $\mathrm{ICH}$. Simultaneously, $\operatorname{Pr} x 1$ increased in astrocytes at the beginning of the $\mathrm{ICH}$, peaked on day 7, and subsequently decreased on day 14. No obvious Prx1 was detected outside the astrocyte. In contrast, the expression of $\operatorname{Pr} x 1$ was further elevated in astrocytes, and the expression of inflammatory factors TNF- $\alpha$ and IL- 6 decreased after treatment with DHI. From these results, it could be concluded that DHI intracellularly promotes the expression of Prx1 after ICH and exerts its neuroprotection though its anti-inflammatory effect. Furthermore, in order to further explore the neuroprotective effect of DHI, Che, an indirect inhibitor of $\operatorname{Pr} x 1$, was chosen to further verify the role of $\operatorname{Pr} x 1$ in ICH. Che promotes the phosphorylation of $\operatorname{Pr} x 1$, which results in the loss of its antioxidant and anti-inflammation effects. These changes lead to the expansion of the hematoma volume, and deterioration in neurological function. DHI treatment cannot reverse the detriments caused by the inactivation of Prx 1 . Taken together, the present results demonstrate the vital role of $\operatorname{Pr} x 1$, and that DHI exerts its neuroprotective effect through the upregulation of $\operatorname{Pr} x 1$.

A previous study indicated that $\operatorname{Pr} x 1$ appeared to act as a sensitive biomarker to ROS (e.g., $\mathrm{H}_{2} \mathrm{O}_{2}$ ) and represented an initial response to stress to maintain redox homeostasis, preventing oxidative damage to lipids and proteins (Scotton et al., 2020). In addition, Prx1 negatively regulated TLR4 signaling for $\mathrm{NF}-\kappa \mathrm{B}$ activation by inhibiting TRAF6 ubiquitin-ligase activity (Min et al., 2018). Furthermore, ROS can activate inflammatory pathways that involve NF$\kappa \mathrm{B}$ signaling, and immune system activation. The production of ROS was orchestrated by inflammatory transcription factors, including nuclear factors derived from erythroid 2

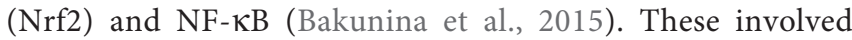
evidences showed that inflammation and oxidative stress are closely correlated. Oxidative stress can mediate inflammation, while inflammation causes damage through oxidative stress. Therefore, it can be concluded that DHI exerts its neuroprotective effect though antioxidative and antiinflammatory functions.

In summary, the present study verified the efficacy of DHI in the treatment of $\mathrm{ICH}$ in the acute phase. DHI exhibited its antioxidative and anti-inflammatory mechanism by upregulating the Prx 1 expression in neurons and astrocytes in a collagenase-induced ICH aged rat model. However, the present study has limitations. One limitation is that merely aged male rats were involved, and the collagenase-induced ICH aged male rat model could not fully simulate the clinical $\mathrm{ICH}$. Another limitation is that DHI is a traditional Chinese medicine, and its ingredients are not fully elucidated. Further studies are needed to differentiate the effective component. Overall, with multiple cellular and molecular targets, DHI exerts its impact on preclinical investigation, and holds therapeutic promise for patients with $\mathrm{ICH}$.

\section{DATA AVAILABILITY STATEMENT}

The datasets generated for this study are available on request to the corresponding authors. 


\section{ETHICS STATEMENT}

The animal study adhered to the institutional guidelines of the Animal Care and Use Committee of Zhengzhou University.

\section{AUTHOR CONTRIBUTIONS}

SW and LY contributed equally to this work, both designed and performed these experiments, drafted and revised the manuscript. HL designed, and supervised the research, and revised the manuscript. GS, YuL, WH, YaL and TP, XW, AS and BQ supervised the research and revised the manuscript. JC provided the magnetic resonance instrument. All authors approved the manuscript.

\section{FUNDING}

This study was supported by grants from the National Natural Science Foundation of China (No. 81971175), the Henan Medical Science and Technology Key Project (Nos. 2018020050 and 2018020094), the Henan Key Special Project for Development and Extension (No. 202102310076), and the Henan Provincial Higher Education Key Research Project Plan (No. 18A320052).

\section{ACKNOWLEDGMENTS}

We would like to thank Jian Wang, Ruhai Huang and Jiarui Wang for the revision of this manuscript.

\section{REFERENCES}

Amenta, F., Bronzetti, E., Sabbatini, M., and Vega, J. A. (1998). Astrocyte changes in aging cerebral cortex and hippocampus: a quantitative immunohistochemical study. Microsc. Res. Tech. 43 (1), 29-33. doi: 10.1002/ (SICI)1097-0029(19981001)43:1<29::AID-JEMT5>3.0.CO;2-H

Bakunina, N., Pariante, C. M., and Zunszain, P.A.J.F.R.B. (2015). Immune mechanisms linked to depression via oxidative stress and neuroprogression. Immunology 144 (3), 365-373. doi: 10.1111/imm.12443

Campuzano, O., Castillo-Ruiz, M. M., Acarin, L., Castellano, B., and Gonzalez, B. (2009). Increased levels of proinflammatory cytokines in the aged rat brain attenuate injury-induced cytokine response after excitotoxic damage. J. Neurosci. Res. 87 (11), 2484-2497. doi: 10.1002/jnr.22074

Castillo-Ruiz, M. M., Campuzano, O., Acarin, L., Castellano, B., and Gonzalez, B. (2007). Delayed neurodegeneration and early astrogliosis after excitotoxicity to the aged brain. Exp. Gerontol. 42 (4), 343-354. doi: 10.1016/j.exger.2006.10.008

Chang, C. F., Cho, S., and Wang, J. (2014). (-)-Epicatechin protects hemorrhagic brain via synergistic Nrf2 pathways. Ann. Clin. Trans. Neurol. 1 (4), 258-271. doi: 10.1002/acn3.54

Chen, Q., Yi, D., Xie, Y., Yang, W., Yang, W., Zhuang, Y., et al. (2011). [Analysis of clinical use of Danhong injection based on hospital information system]. Zhongguo Zhong yao za zhi= Zhongguo zhongyao zazhi = China J. Chin. Mater. Med. 36 (20), 2817-2820. doi: 10.1111/ j.1744-7909.2011.01037.x

Cordonnier, C., Demchuk, A., Ziai, W., and Anderson, C. S. (2018). Intracerebral haemorrhage: current approaches to acute management. Lancet (London England) 392 (10154), 1257-1268. doi: 10.1016/S01406736(18)31878-6

Del Bigio, M. R., Yan, H. J., Buist, R., and Peeling, J. (1996). Experimental intracerebral hemorrhage in rats. Magnetic resonance imaging and histopathological correlates. Stroke 27 (12), 2312-9; discussion 2319-20. doi: 10.1161/01.STR.27.12.2312

Feng, X., Li, Y., Wang, Y., Li, L., Little, P. J., Xu, S. W., et al. (2019). Danhong injection in cardiovascular and cerebrovascular diseases: Pharmacological actions, molecular mechanisms, and therapeutic potential. Pharmacol. Res. 139, 62-75. doi: 10.1016/j.phrs.2018.11.006

Guan, Y., Yin, Y., Zhu, Y. R., Guo, C., Wei, G., Duan, J. L., et al. (2013). Dissection of mechanisms of a chinese medicinal formula: danhong injection therapy for myocardial ischemia/reperfusion injury in vivo and in vitro. Evidence-Based Complement. Altern. Med. : eCAM 2013, 972370. doi: 10.1111/j.1744-7909.2011.01037.x
Guo, H., Li, M. J., Liu, Q. Q., Guo, L. L., Ma, M. M., Wang, S. X., et al. (2014). Danhong injection attenuates ischemia/reperfusion-induced brain damage which is associating with Nrf2 levels in vivo and in vitro. Neurochem. Res. 39 (9), 1817-1824. doi: 10.1007/s11064-014-1384-1

Guo, Z. L., Zhu, Y., Su, X. T., Liu, J., Yang, Q. X., Nan, J. Y., et al. (2015). DanHong injection dose-dependently varies amino acid metabolites and metabolic pathways in the treatment of rats with cerebral ischemia. Acta Pharmacol. Sin. 36 (6), 748-757. doi: 10.1038/aps.2014.167

Han, X., Lan, X., Li, Q., Gao, Y., Zhu, W., Cheng, T., et al. (2016). Inhibition of prostaglandin E2 receptor EP3 mitigates thrombin-induced brain injury. J. Cereb. Blood Flow Metab. : Off. J. Int. Soc. Cereb. Blood Flow Metab. 36 (6), 1059-1074. doi: 10.1177/0271678X15606462

Han, X., Zhao, X., Lan, X., Li, Q., Gao, Y., Liu, X., et al. (2019). 20-HETE synthesis inhibition promotes cerebral protection after intracerebral hemorrhage without inhibiting angiogenesis. J. Cerebr. Blood F Met. 39 (8), 1531-1543. doi: $10.1177 / 0271678 X 18762645$

He, Y., Wan, H., Du, Y., Bie, X., Zhao, T., Fu, W., et al. (2012). Protective effect of Danhong injection on cerebral ischemia-reperfusion injury in rats. $J$. Ethnopharmacol. 144 (2), 387-394. doi: 10.1016/j.jep.2012.09.025

Hemphill, J. C.3rd, Adeoye, O. M., Alexander, D. N., Alexandrov, A. W., Amin-Hanjani, S., Cushman, M., et al. (2018). Clinical Performance Measures for Adults Hospitalized With Intracerebral Hemorrhage: Performance Measures for Healthcare Professionals From the American Heart Association/American Stroke Association. Stroke. 49 (7) e243-e261. doi: 10.1161/STR.0000000000000171

Hu, X., Tao, C., Gan, Q., Zheng, J., Li, H., and You, C. (2016). Oxidative Stress in Intracerebral Hemorrhage: Sources, Mechanisms, and Therapeutic Targets. Oxid. Med. Cell. Longevity 2016, 3215391. doi: 10.1155/2016/3215391

Ironside, N., Chen, C. J., Ding, D., Mayer, S. A., and Connolly, E. S.Jr. (2019). Perihematomal Edema After Spontaneous Intracerebral Hemorrhage. Stroke 50 (6), 1626-1633. doi: 10.1161/STROKEAHA.119.024965

Jeong, S. J., Kim, S., Park, J. G., Jung, I. H., Lee, M. N., Jeon, S., et al. (2018). Prdx1 (peroxiredoxin 1) deficiency reduces cholesterol efflux via impaired macrophage lipophagic flux. Autophagy 14 (1), 120-133. doi: 10.1080/ 15548627.2017.1327942

Jiang, X., Lv, B., Li, P., Ma, X., Wang, T., Zhou, Q., et al. (2015). Bioactivityintegrated UPLC/Q-TOF-MS of Danhong injection to identify NF-kappaB inhibitors and anti-inflammatory targets based on endothelial cell culture and network pharmacology. J. Ethnopharmacol. 174, 270-276. doi: 10.1016/j.jep.2015.08.026

Lan, X., Han, X., Li, Q., Li, Q., Gao, Y., Cheng, T., et al. (2017). Pinocembrin protects hemorrhagic brain primarily by inhibiting toll-like receptor 4 and 
reducing M1 phenotype microglia. Brain Behav. Immun. 61, 326-339. doi: 10.1016/j.bbi.2016.12.012

Ledgerwood, E. C., Marshall, J. W., and Weijman, J. F. (2017). The role of peroxiredoxin 1 in redox sensing and transducing. Arch. Biochem. Biophys. 617, 60-67. doi: 10.1016/j.abb.2016.10.009

Lekic, T., Rolland, W., Manaenko, A., Fathali, N., and Zhang, J. H. (2012). "Corner Turning Test for Evaluation of Asymmetry After Intracerebral Hemorrhage, in Animal Models of Acute Neurological Injuries II (Rodents Humana Press).

Li, P., Su, W., Yun, S., Liao, Y., Liao, Y., Liu, H., et al. (2017). Toward a scientific understanding of the effectiveness, material basis and prescription compatibility of a Chinese herbal formula Dan-hong injection. Sci. Rep. 7, 46266. doi: 10.1038/srep46266

Li, Q., Wan, J., Lan, X., Han, X., Wang, Z., and Wang, J. (2017). Neuroprotection of brain-permeable iron chelator VK-28 against intracerebral hemorrhage in mice. J. Cereb. Blood Flow Metab. : Off. J. Int. Soc. Cereb. Blood Flow Metab. 37 (9), 3110-3123. doi: 10.1177/0271678X17709186

Liu, X., Wu, Z., Yang, K., Ding, H., and Wu, Y. (2013). Quantitative analysis combined with chromatographic fingerprint for comprehensive evaluation of Danhong injection using HPLC-DAD. J. Pharm. Biomed. Anal. 76, 70-74. doi: 10.1016/j.jpba.2012.12.013

Liu, D. L., Zhao, L. X., Zhang, S., and Du, J. R. (2016). Peroxiredoxin 1-mediated activation of TLR4/NF-kappaB pathway contributes to neuroinflammatory injury in intracerebral hemorrhage. Int. Immunopharmacol. 41, 82-89. doi: 10.1016/j.intimp.2016.10.025

Lu, Y., Zhang, X. S., Zhou, X. M., Gao, Y. Y., Chen, C. L., Liu, J. P., et al. (2019). Peroxiredoxin $1 / 2$ protects brain against $\mathrm{H} 2 \mathrm{O} 2$-induced apoptosis after subarachnoid hemorrhage. FASEB J. : Off. Publ. Fed. Am. Soc. Exp. Biol. 33 (2), 3051-3062. doi: 10.1096/fj.201801150R

Lyu, M., Yan, C. L., Liu, H. X., Wang, T. Y., Shi, X. H., Liu, J. P., et al. (2017). Network pharmacology exploration reveals endothelial inflammation as a common mechanism for stroke and coronary artery disease treatment of Danhong injection. Sci. Rep. 7 (1), 15427. doi: 10.1038/s41598-01714692-3

Masoro, E. J. (1980). Mortality and growth characteristics of rat strains commonly used in aging research. Exp. Aging Res. 6 (3), 219-233. doi: 10.1080/ 03610738008258359

Min, Y., Mi-Jeong, K., Sena, L., Eunyoung, C., and Autophagy, L.K.-Y.J. (2018). Inhibition of TRAF6 ubiquitin-ligase activity by PRDX1 leads to inhibition of NFKB activation and autophagy activation. Autophagy 14 (8), 1347-1358. doi: 10.1080/15548627.2018.1474995

Mizusawa, H., Ishii, T., Bannai, S., and Peroxiredoxin, I. (2000). (macrophage 23 $\mathrm{kDa}$ stress protein) is highly and widely expressed in the rat nervous system. Neurosci. Lett. 283 (1), 57-60. doi: 10.1016/S0304-3940(00)00910-1

Monti, B., Virgili, M., and Contestabile, A. (2004). Alterations of markers related to synaptic function in aging rat brain, in normal conditions or under conditions of long-term dietary manipulation. Neurochem. Int. 44 (8), 579584. doi: 10.1016/j.neuint.2003.10.007

Nakaso, K., Kitayama, M., Mizuta, E., Fukuda, H., Ishii, T., Nakashima, K., et al. (2000). Co-induction of heme oxygenase- 1 and peroxiredoxin I in astrocytes and microglia around hemorrhagic region in the rat brain. Neurosci. Lett. 293 (1), 49-52. doi: 10.1016/S0304-3940(00)01491-9

Perkins, A., Nelson, K. J., Parsonage, D., Poole, L. B., and Karplus, P. A. (2015). Peroxiredoxins: guardians against oxidative stress and modulators of peroxide signaling. Trends Biochem. Sci. 40 (8), 435445. doi: 10.1016/j.tibs.2015.05.001

Qian, J., Zhao, X., Wang, W., Zhang, S., Hong, Z., Chen, X., et al. (2018). Transcriptomic Study Reveals Recovery of Impaired Astrocytes Contribute to Neuroprotective Effects of Danhong Injection Against Cerebral Ischemia/ Reperfusion-Induced Injury. Front. Pharmacol. 9, 250. doi: 10.3389/ fphar.2018.00250

Rawat, S. J., Creasy, C. L., Peterson, J. R., and Chernoff, J. (2013). The tumor suppressor Mst1 promotes changes in the cellular redox state by phosphorylation and inactivation of peroxiredoxin-1 protein. J. Biol. Chem. 288 (12), 8762-8771. doi: 10.1074/jbc.M112.414524
Ren, C., Guingab-Cagmat, J., Kobeissy, F., Zoltewicz, S., Mondello, S., Gao, M., et al. (2014). A neuroproteomic and systems biology analysis of rat brain post intracerebral hemorrhagic stroke. Brain Res. Bull. 102, 46-56. doi: 10.1016/ j.brainresbull.2014.02.005

Rhee, S. G., Kang, S. W., Jeong, W., Chang, T. S., Yang, K. S., and Woo, H. A. (2005). Intracellular messenger function of hydrogen peroxide and its regulation by peroxiredoxins. Curr. Opin. Cell Biol. 17 (2), 183-189. doi: 10.1016/j.ceb.2005.02.004

Rosenberg, G. A., Mun-Bryce, S., Wesley, M., and Kornfeld, M. (1990). Collagenase-induced intracerebral hemorrhage in rats. Stroke 21 (5), 801807. doi: 10.1161/01.STR.21.5.801

Sacchetti, B., and Bielavska, E. (1998). Chelerythrine, a specific PKC inhibitor, blocks acquisition but not consolidation and retrieval of conditioned taste aversion in rat. Brain Res. 799 (1), 84-90. doi: 10.1016/S0006-8993(98) 00460-0

Schaar, K. L., Brenneman, M. M., and Savitz, S. I. (2010). Functional assessments in the rodent stroke model. Exp. Trans. Stroke Med. 2 (1), 13. doi: 10.1186/2040-7378-2-13

Scotton, E., Colombo, R., Reis, J. C., Possebon, G. M. P., Hizo, G. H., Valiati, F E., et al. (2020). BDNF prevents central oxidative damage in a chronic unpredictable mild stress model: The possible role of PRDX-1 in anhedonic behavior. Behav. Brain Res. 378, 112245. doi: 10.1016/ j.bbr.2019.112245

SFDA. (2002). National Drugs Surveillance Administrative Bureau Standard WS11220 (ZD-1220)-2002.

Sun, M., Zhang, J. J., Shan, J. Z., Zhang, H., Jin, C. Y., Xu, S., et al. (2009). Clinical observation of Danhong Injection (herbal TCM product from Radix Salviae miltiorrhizae and Flos Carthami tinctorii) in the treatment of traumatic intracranial hematoma. Phytomed. : Int. J. Phytother. Phytopharm. 16 (8), 683-689. doi: 10.1016/j.phymed.2009.03.020

Wang, Y., Jiang, Z., Yang, F., Chai, X., Zhu, Y., Zhao, X., et al. (2016). Establishment of a ternary network system for evaluating the antioxidant fraction of Danhong injection. Biomed. Chromatogr. : BMC 30 (10), 16661675. doi: $10.1002 / \mathrm{bmc} .3739$

Wang, H., Ren, S., Liu, C., and Zhang, X. (2016). An Overview of Systematic Reviews of Danhong Injection for Ischemic Stroke. Evidence-Based Complement. Altern. Med. : eCAM 2016, 8949835. doi: 10.1155/2016/ 8949835

Wang, Z., Zhou, F., Dou, Y., Tian, X., Liu, C., Li, H., et al. (2018). Melatonin Alleviates Intracerebral Hemorrhage-Induced Secondary Brain Injury in Rats via Suppressing Apoptosis, Inflammation, Oxidative Stress, DNA Damage, and Mitochondria Injury. Trans. Stroke Res. 9 (1), 74-91. doi: 10.1007/s12975-017-0559-x

Wang, G., Wang, L., Sun, X. G., and Tang, J. (2018). Haematoma scavenging in intracerebral haemorrhage: from mechanisms to the clinic. J. Cell. Mol. Med. 22 (2), 768-777. doi: $10.1111 / \mathrm{jcmm} \cdot 13441$

Wang, J. (2010). Preclinical and clinical research on inflammation after intracerebral hemorrhage. Prog. Neurobiol. 92 (4), 463-477. doi: 10.1016/j.pneurobio.2010.08.001

Wu, H., Wu, T., Han, X., Wan, J., Jiang, C., Chen, W., et al. (2017). Cerebroprotection by the neuronal PGE2 receptor EP2 after intracerebral hemorrhage in middle-aged mice. J. Cereb. Blood Flow Metab. : Off. J. Int. Soc. Cereb. Blood Flow Metab. 37 (1), 39-51. doi: 10.1177/ 0271678X15625351

Yamamoto, S., Yang, G., Zablocki, D., Liu, J., Hong, C., Kim, S. J., et al. (2003). Activation of Mst1 causes dilated cardiomyopathy by stimulating apoptosis without compensatory ventricular myocyte hypertrophy. J. Clin. Invest. 111 (10), 1463-1474. doi: 10.1172/JCI17459

Yang, J., Li, Q., Wang, Z., Qi, C., Han, X., Lan, X., et al. (2017). Multimodality MRI assessment of grey and white matter injury and blood-brain barrier disruption after intracerebral haemorrhage in mice. Sci. Rep. 7, 40358. doi: $10.1038 /$ srep 40358

Zhang, Y. Y., Zhou, H. F., Yang, J. H., He, Y., Chen, X. Q., Nishinari, K., et al. (2016). Effects of Danhong Injection () and its main components on anticoagulation and fibrinolysis in cultured vein endothelial cells. Chin. J. Integr. Med. 22 (4), 276-283. doi: 10.1007/s11655-016-2498-x 
Zhang, Z., Song, Y., Zhang, Z., Li, D., Zhu, H., Liang, R., et al. (2017). Distinct role of heme oxygenase-1 in early- and late-stage intracerebral hemorrhage in 12month-old mice. J. Cereb. Blood Flow Metab. : Off. J. Int. Soc. Cereb. Blood Flow Metab. 37 (1), 25-38. doi: 10.1177/0271678X16655814

Zhao, X., Wu, T., Chang, C. F., Wu, H., Han, X., Li, Q., et al. (2015). Toxic role of prostaglandin E2 receptor EP1 after intracerebral hemorrhage in mice. Brain Behav. Immun. 46, 293-310. doi: 10.1016/j.bbi.2015.02.011

Zhu, W., Gao, Y., Wan, J., Lan, X., Han, X., Zhu, S., et al. (2018). Changes in motor function, cognition, and emotion-related behavior after right hemispheric intracerebral hemorrhage in various brain regions of mouse. Brain Behav. Immun. 69, 568-581. doi: 10.1016/j.bbi.2018.02.004
Conflict of Interest: The authors declare that the research was conducted in the absence of any commercial or financial relationships that could be construed as a potential conflict of interest.

Copyright (C) 2020 Wang, Yu, Sun, Liu, Hu, Liu, Peng, Wang, Cheng, Sr, Qin and Lu. This is an open-access article distributed under the terms of the Creative Commons Attribution License (CC BY). The use, distribution or reproduction in other forums is permitted, provided the original author(s) and the copyright owner(s) are credited and that the original publication in this journal is cited, in accordance with accepted academic practice. No use, distribution or reproduction is permitted which does not comply with these terms. 